\title{
Dynamic Project and Workflow Management for Design Processes in Chemical Engineering
}

\author{
Markus Heller ${ }^{\mathrm{a}}$, Bernhard Westfechtel ${ }^{\mathrm{a}}$

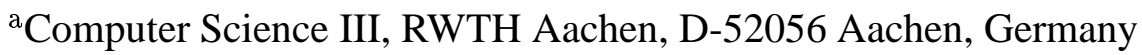

\begin{abstract}
Design processes in chemical engineering are hard to support. The design process is highly creative, many design alternatives are explored, and both unexpected and planned feedback occurs frequently. Thus, it is inherently difficult to manage the workflow in design processes, i.e., to coordinate the effort of experts working on tasks such as creation of flow diagrams, steady-state and dynamic simulations, etc. Conventional project and workflow management systems support the management of design processes only to a limited extent. In contrast, the management system AHEAD is designed specifically for dynamic design processes.
\end{abstract}

Keywords process systems engineering, business decision making, workflow management

\section{INTRODUCTION}

Design processes in chemical engineering are hard to support. Since design processes are highly creative, they can rarely be planned completely in advance. Rather, planning and execution may have to be interleaved seamlessly. In the course of the design process, many design alternatives are explored which are mutually dependent. Furthermore, design proceeds iteratively, starting from sketchy, coarse-level designs to detailed designs which are eventually needed for building the respective chemical plant. Iterations may cause feedback to earlier steps of the design process; it may be necessary to revoke inadequate design decisions. Finally, design involves cooperation among team members from different disciplines and potentially multiple enterprises, causing additional difficulties concerning the coordination of the overall design process.

Technical tools such as flowsheet editors, simulators for steady-state and dynamic simulations, etc. are crucial aids for effectively and efficiently performing design tasks. In addition, managerial tools are required which address the coordination of design processes. In fact, such tools are crucial for supporting business decision making. In the course of the design process, many decisions have to be made concerning the steps of the chemical process, the relationships among these steps, the realization of chemical process steps by devices, etc. To perform these decisions, design alternatives have to be identified and elaborated, and the respective design tasks have to be coordinated regarding their mutual interfaces and dependencies. To support business decision making, managerial tools must provide chief designers with accurate views of the design process at an adequate level of granularity, offer tools for planning, controlling, and coordinating design tasks, thereby taking care of the dynamics of design processes.

Currently, many management tools are available which, unfortunately, often have not been developed for supporting complex and dynamic design processes. In fact, most of these tools are generic in the sense that they can be applied to any kind of business process (there are a few process support tools designed for chemical engineering such as e.g. n-dim [1] and KBDS [2]). For example, project management systems [3] assist managers in project planning and control, assuming that the project can be represented by a partially ordered set of activities. However, iteration and feedback cannot be represented in project plans. Furthermore, important 
information is missing concerning e.g. the products of design tasks such as e.g. different kinds of flow sheets and simulation models. To some extent, project management systems are useful for high-level planning and control at the level of milestones. But to support decision making effectively, other sources of information have to be exploited, as well.

Workflow management systems [4] have been developed for supporting routine processes performed e.g. in banks, insurance companies, administrations, etc. A workflow management system manages the flow of work between participants, according to a defined procedure consisting of a number of tasks. It coordinates user and system participants to achieve defined objectives by set deadlines. To this end, tasks and documents are passed from participant to participant in a correct order. Moreover, a workflow management system may offer an interface to invoke a tool on a document either interactively or automatically. Workflow management systems differ from project management systems since they address rather detailed execution support rather than only high-level planning. Their most important restriction is limited support for dynamic design processes. Many workflow management systems assume a statically defined workflow that cannot be changed during execution. This assumption does not match the characteristics of design processes, which are highly creative, dynamic, and iterative, and therefore cannot be controlled by a static workflow defined in advance. Though this problem has been recognized in the workflow community [5], so-called adaptive workflow management systems provide at best partial solutions (e.g., based on exceptions, which, however, have to be pre-defined). Still, many of the commercial tools hardly address the problem of evolving workflows.

In this paper, we present a management system which has been developed to support engineering design processes. This system is called AHEAD (Adaptable and Human-Centered Environment for the MAnagement of Design Processes [6, 7]). It has been developed in the context of the long-term research project IMPROVE [8] which is concerned with models and tools for design processes in chemical engineering. AHEAD equally covers products, activities, and resources and therefore offers more comprehensive support than project or workflow management systems. Moreover, AHEAD supports seamless interleaving of planning and execution - a crucial requirement which workflow management systems usually do not meet. Design processes are represented by dynamic task nets, which may evolve continuously throughout the execution of a design process. Dynamic task nets include modeling elements specifically introduced for design processes, e.g., feedback relationships for iterations in the design process which cannot be represented in project plans. This way, AHEAD improves business decision making since it offers a more natural, realistic, and adequate representation of design processes.

\section{SYSTEM DESCRIPTION}

Figure 1 gives an overview of the AHEAD system. AHEAD offers environments for different kinds of users, which are called modeler, manager, and designer. Please note that "modeler", "manager", and "designer" denote roles rather than persons. In particular, one person may play multiple roles. For example, the chief designer of a project may act both as a manager and as a designer. Similarly, management and modeling may be performed by the same person.

The management environment supports project managers in planning, analyzing, monitoring, and controlling design processes. In particular, planning and execution may be interleaved seamlessly so that the dynamics of design processes may be taken into account. Since the management environment is coupled with the work environments for designers, the manager is provided with accurate and current information about the state of his project. Management comprises activities - the design tasks and their relationships, products - the design doc- 


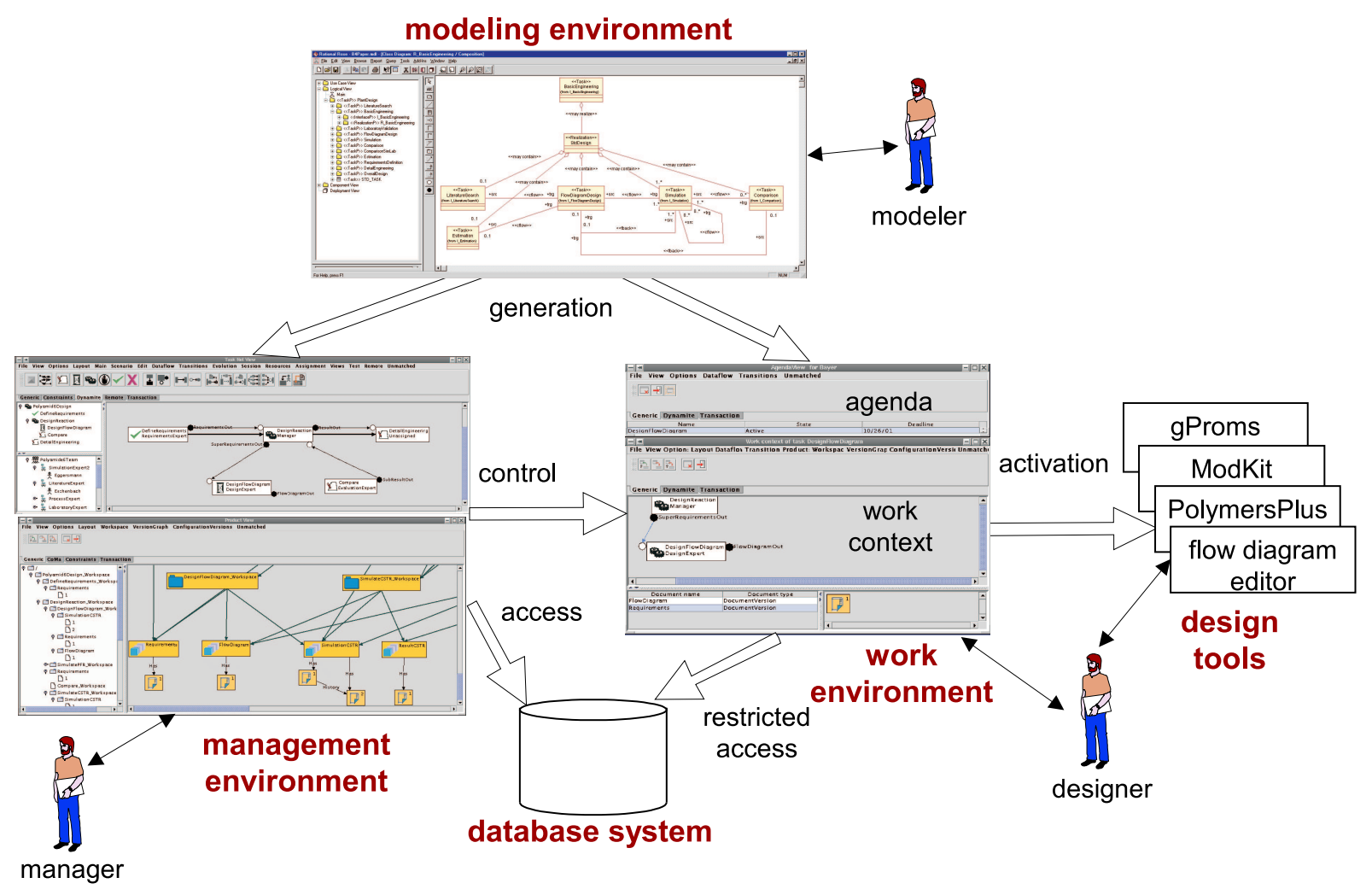

Figure 1. Architecture of the AHEAD system.

uments and their dependencies, and resources - both human resources, i.e., the design team, and technical resources, i.e., the software tools used by the team. The management environment offers different kinds of graphical representations, e.g., trees, diagrams, and tables.

The work environment supports designers in their work as far as the coordination aspects are concerned. Management and work environments are integrated through a common database which stores a graph representation of the respective design process. The work environment provides an agenda tool which displays the tasks assigned to a designer in a table containing information about state, deadline, expected duration, etc. The designer may perform operations such as starting, suspending, finishing, or aborting a task. Furthermore, he may open a work context which manages the documents and tools required for executing a certain task. The designer is supplied with a workspace of versioned documents. He may work on a document by starting a tool such as e.g. a flow diagram editor, a simulation tool, etc. The workspace may change dynamically during task execution (which may take hours, days, or weeks rather than minutes); e.g., a new version of some input document may arrive during task execution.

Management and work environment are used at project run-time to execute design processes. The modeling environment allows incorporation of domain-specific knowledge about design processes into the AHEAD system. Domain knowledge is expressed in the Unified Modeling Language. With the help of class diagrams, design processes may be modeled on the type level. For example, task classes may be defined for designing flow sheets, performing steady-state or dynamic simulations, etc. In addition, recurring patterns of task instances may be defined with the help of collaboration diagrams. Using the UML model, process support may be tailored towards the respective domain. These models may evolve during execution, as well [9]. 


\section{APPLICATION}

In the IMPROVE project, we are studying the design of a chemical plant for Polyamide 6 as a reference process against which the developed tools (e.g., AHEAD) are evaluated. The process focuses on the early lifecycle phases, namely basic engineering and conceptual design. It is based on interviews conducted with an industrial partner, study of literature, and own research. To some extent, the reference process reflects current industrial practice, but it also contributes innovative aspects such as simultaneous engineering and inter-organizational cooperation.

For a comprehensive description of the reference process, see [10]. Here, we focus on the managerial parts of this process, which are supported by the AHEAD system. We illustrate this process support by discussing the task net displayed in Figure 2. This task net evolves during execution, but this evolution is not shown graphically due to the lack of space.

Task nets are shown to the user of the management environment of AHEAD in roughly the same way as in the figure. Tasks are represented by rectangles. Each task may have inputs and outputs shown as white and black circles, respectively. Tasks are connected by control flows which resemble precedence relationships in project plans. Feedback flows represent feedback in the design process and are oriented oppositely to control flows. Data flows connect task outputs and inputs. Hierarchical relationships are not shown explicitly in the figure; rather, they are expressed by placing subtasks below their supertasks.

At the start of the design process, the manager creates an initial task net comprising only the task Preparation and its refinements. In the preparation phase, the requirements to the chemical plant are defined, a literature research is carried out, and an initial abstract flow diagram is created. Based on this information, the alternatives batch and continuous operation are compared, and a decision is performed towards continuous operation. The result of the preparation phase is an abstract flow diagram, according to which the design process may be detailed further. To this end, the manager extends the task net with tasks for designing the reaction, the separation, and the compounding (using an extruder), which are the major steps of the respective chemical process. To design the reaction, a process flow diagram is created which defines alternatives for this part of the chemical process. Only then may the task net be extended with design tasks for elaborating these alternatives (a CSTR reactor, a PFR reactor, or two reactor cascades). Simulations are carried out to evaluate these alternatives; if necessary, laboratory experiments are performed to validate the simulation models. Finally, a decision is performed with respect to the selected alternative (task Compare). The subnet for designing the separation is structured in a similar way; here, the alternatives extraction and evaporation are considered. To start the investigation of the separation step as soon as possible, an initial estimate of the output of the reaction step is made (simultaneous engineering). The subnet for designing the extruder is structured in a different way; special-purpose simulation tools are used to this end. Please note the feedback flow from DesignExtruder to DesignSeparation: it is not clear from the beginning to what extent the extruder can be used for the separation of remaining input substances. This requires negotiation between the designer of the separation step and the designer of the extruder. After having designed all parts of the chemical process, the overall concept is jointly discussed in a final decision step.

\section{CONCLUSION}

The sample process sketched above shows how managers and designers are supported in making business decisions concerning the plant design. It shows the typical structure of the design process: elaborate alternatives, evaluate them by simulations, laboratory experiments, and cost 


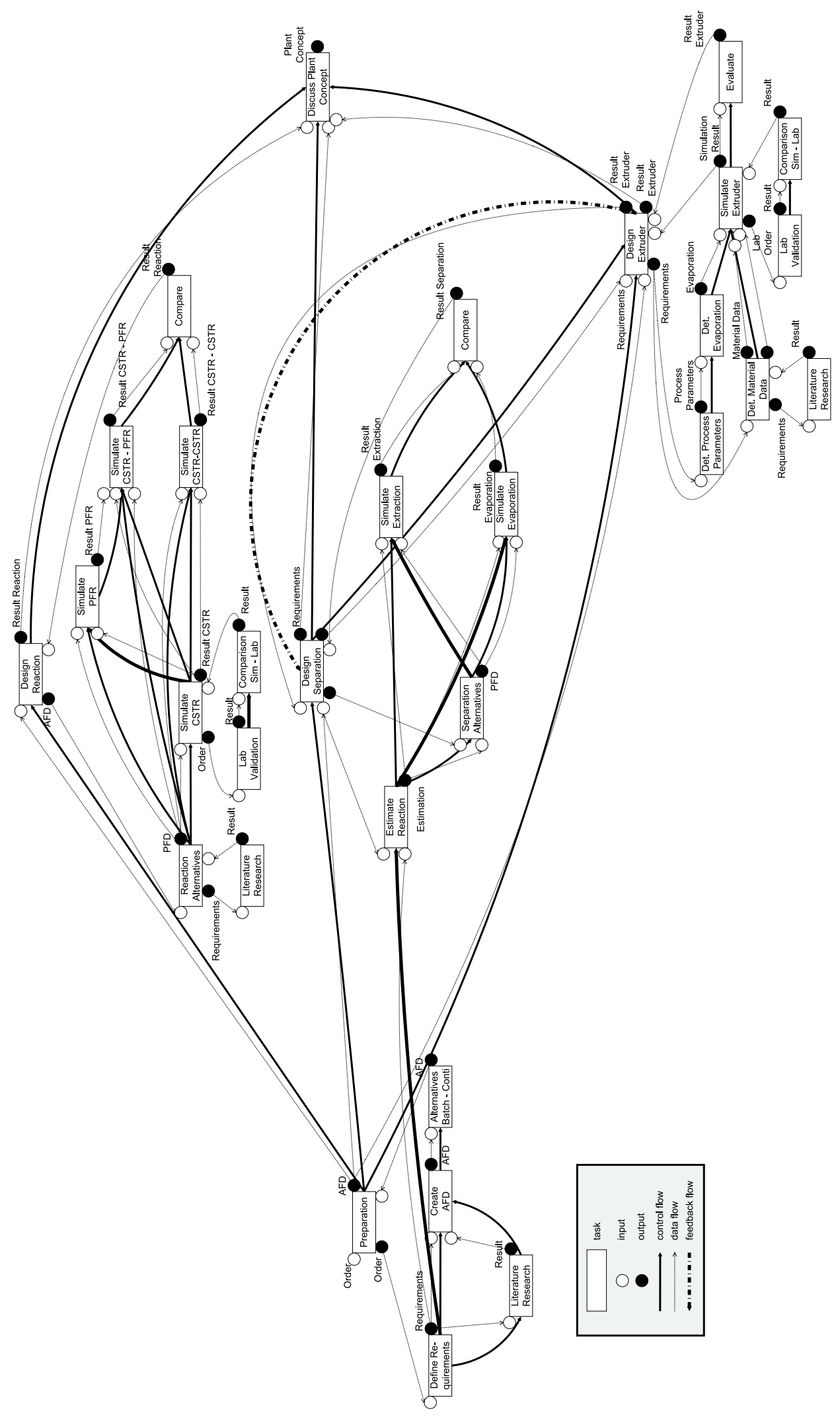

Figure 2. Task net for the polyamide6 design process. 
calculations, select an alternative, and proceed by iterative refinement of the design. Decision steps are explicitly modeled as tasks. The overall task net evolves during execution. Different aspects of dynamics are taken into account, such as product-dependent task nets, simultaneous engineering, and feedback. Please note that managerial and technical activities are tightly integrated; this is necessary to provide effective decision support.

Business decision making of this kind cannot be provided by conventional project and workflow management systems. In a project management system, the manager may define milestones based on a simple conceptual model of partially ordered activities. But project plans do not reflect well the characteristics of highly dynamic, iterative, and creative design processes. Furthermore, the link to the actual design activities is missing. Workflow management systems would typically constrain the design process very tightly and tend to automate the design process too far. The approach realized in the AHEAD system provides the required flexibility and promises more adequate decision support at the managerial level.

\section{REFERENCES}

[1] Arthur W. Westerberg, Eswaran Subrahmanian, Yoram Reich, Suresh Konda, and the ndim group. Designing the process design process. Computers \& Chemical Engineering, 21(S):S1-S9, 1997.

[2] R. Bañares-Alcántara and H.M.S. Lababidi. Design support systems for process engineering - II. KBDS: An experimental prototype. Computers \& Chemical Engineering, 19(3):279-301, 1995.

[3] Harold Kerzner. Project Management: A Systems Approach to Planning, Scheduling, and Controlling. John Wiley \& Sons, New York, 1998.

[4] Peter Lawrence, editor. Workflow Handbook. John Wiley \& Sons, Chichester, UK, 1997.

[5] Dimitrios Georgakopoulos, Wolfgang Prinz, and Alexander L. Wolf, editors. Proceedings of the International Joint Conference on Work Activities Coordination and Collaboration (WACC-99), volume 24-2 of ACM SIGSOFT Software Engineering Notes, San Francisco, CA, 1999. ACM Press.

[6] Dirk Jäger, Ansgar Schleicher, and Bernhard Westfechtel. AHEAD: A graph-based system for modeling and managing development processes. In Manfred Nagl, Andy Schürr, and Manfred Münch, editors, AGTIVE - Applications of Graph Transformations with Industrial Relevance, LNCS 1779, pages 325-339, Castle Rolduc, The Netherlands, 1999. Springer-Verlag.

[7] Manfred Nagl, Bernhard Westfechtel, and Ralph Schneider. Tool support for the management of design processes in chemical engineering. Computers \& Chemical Engineering, 27(2):175-197, 2003.

[8] Manfred Nagl and Wolfgang Marquardt. SFB-476 IMPROVE: Informatische Unterstützung übergreifender Entwicklungsprozesse in der Verfahrenstechnik. In Matthias Jarke, Klaus Pasedach, and Klaus Pohl, editors, Informatik '97: Informatik als Innovationsmotor, Informatik aktuell, pages 143-154, Aachen, Germany, September 1997. Springer-Verlag.

[9] Ansgar Schleicher. Management of Development Processes - An Evolutionary Approach. Deutscher Universitäts-Verlag, Wiesbaden, Germany, 2002.

[10] B. Bayer, M. Eggersmann, R. Gani, and R. Schneider. Case studies in process design. In B. Braunschweig and R. Gani, editors, Software Architectures and Tools for Computer Aided Process Engineering. Elsevier Publishers, 2002. 